\title{
Avaliação do efeito da combinação de pectina, gelatina e alginato de sódio sobre as características de gel de fruta estruturada a partir de "mix" de polpa de cajá e mamão, por meio da metodologia de superfície de resposta
}

\author{
Ana Vania CARVALHO ${ }^{1}$, Rafaella Andrade MATTIETTO², Glaucy Takeda ASSIS 3 , \\ Lúcia Fátima Henriques LOURENÇO ${ }^{4}$
}

\section{RESUMO}

Este trabalho teve por objetivo estabelecer procedimento tecnológico para produção de fruta estruturada a partir de "mix" de polpa de cajá e mamão, procurando unir as propriedades sensoriais de cada uma das frutas e potencializar as características funcionais do produto final. Avaliou-se o efeito da combinação de pectina, gelatina e alginato de sódio, via metodologia de superfície de resposta, nas características do gel de fruta. As polpas de mamão e cajá e os estruturados obtidos foram caracterizados com relaçáo aos compostos funcionais, avaliando-se o teor de taninos e carotenóides totais, além das análises de composição centesimal, $\mathrm{pH}$, acidez titulável, sólidos solúveis, açúcares, atividade de água, carboidratos e valor energético total. Os resultados obtidos através do planejamento experimental indicam que para o estruturado misto de cajá e mamão, somente o aumento da concentração de gelatina afeta a firmeza do produto final. Os estruturados de frutas desenvolvidos apresentaram boa aceitaçáo sensorial para todos os atributos avaliados. Com relaçáo à intençáo de compra, $70 \%$ dos provadores responderam que provavelmente ou certamente, comprariam o estruturado misto de cajá e mamão se o encontrassem à venda. PALAVRAS-CHAVE: fruta estruturada; substâncias funcionais; planejamento experimental; sensorial.

\section{Evaluation of the effects of pectin, gelatin and sodium alginate combination on the characteristics of a mixed structured fruit gel from papaya and yellow mobin, by response surface methodology}

\section{ABSTRACT}

The objective of this work was to establish a technological procedure for producing a structured fruit product from yellow mobin and papaya purees, as an attempt to combine the sensory properties of both fruits and potentialize the functional properties of the final product. The effect of different combinations of pectin, gelatin and sodium alginate on the fruit gel properties was evaluated by response surface methodology. Papaya and yellow mobin purees and the structured products were characterized in terms of functional compounds (total tannin and carotenoid contents), as well as centesimal composition, $\mathrm{pH}$, titratable acidity, soluble solids, sugars, water activity, carbohydrates and total energetic value. The results obtained from the experimental design indicated that only the gelatin concentration affected the product firmness. The developed fruit structured products presented good acceptance for all evaluated attributes. The data concerning purchase intent indicated that $70 \%$ of the panelists would probably or certainly purchase the mixed yellow mobin and papaya structured product if they found it for sale. KEYWORDS: structured fruit; functional compounds; experimental design; sensory analysis.

\footnotetext{
1 Embrapa Amazônia Oriental. anavania@cpatu.embrapa.br

2 Embrapa Amazônia Oriental. rafaella@cpatu.embrapa.br

3 glaucy_takeda@hotmail.com

${ }^{4}$ Universidade Federal do Pará. lucia@ufpa.br
} 


\section{INTRODUÇÃO}

Hoje, no mercado norte americano, produtos como o estruturado de frutas, encontram-se já bem estabelecidos. O Brasil, ao contrário, ainda apresenta um amplo e promissor mercado a ser explorado em relação a tais tipos de produtos, principalmente pela grande riqueza de sabores encontrados em todo o seu território com relaçáo às frutas tropicais. Estruturados de frutas são produtos obtidos do purê de frutas, devidamente formulados para a obtenção de produtos nutritivos, com boa textura e sabor. São utilizados hidrocolóides, responsáveis pela redução da umidade do alimento e estruturação da polpa, por meio de uma gelatinização, proporcionando textura e aspecto agradáveis ao produto final. Os produtos depois de estruturados são submetidos à secagem, apresentando boa estabilidade (Morley e Sharma 1986; Grizotto et al. 2005a).

O cajá (Spondias lutea L.), fruto originário de árvore frutífera tropical e perene, apresenta grande aceitação no mercado, sendo considerado bastante saboroso e nutritivo. Trata-se de espécie que sobrevive de forma silvestre, cuja exploração é feita de modo extrativista. Além da importância regional, o cajá vem ganhando papel de destaque no agronegócio brasileiro, com o desenvolvimento de novos produtos e a comercialização em maior escala de sua polpa (Bosco et al. 2000; Sacramento e Sousa 2000).

$\mathrm{O}$ fruto apresenta elevado teor de carotenóides, que dão à sua polpa, além de uma intensa coloração amarela, um apelo funcional bastante significativo (Mattietto 2005). O cajá também é um fruto rico em taninos. A elevada associação entre o consumo de alimentos vegetais ricos em taninos e a menor incidência de determinados tipos de doenças crônicodegenerativas, observados em vários grupos populacionais, tem estimulado a pesquisa científica a explorar possíveis propriedades funcionais dessas substâncias no organismo humano (Tirapegui e Castro 2001).

O mamão (Carica papaya) é disponível para o consumo ao longo de todo o ano e tem boa aceitabilidade entre crianças e adultos. É considerado um fruto com boas características sensoriais e nutricionais, apresentando teores consideráveis de carotenóides totais, vitamina C, sais minerais e carboidratos (Sentanin e Amaya 2007).

De sabores exóticos e agradáveis ao paladar, o cajá e o mamão proporcionam uma interessante combinação de sabor e saúde. Assim, o objetivo deste trabalho foi investigar os parâmetros relevantes para a otimização do processo de estruturação do "mix" de polpa de cajá e mamão, avaliando-se o efeito da combinaçáo dos hidrocolóides: gelatina, pectina e alginato de sódio, na firmeza, sólidos solúveis, $\mathrm{pH}$ e atividade de água do gel de fruta, além de caracterizar o produto final quanto aos seus atributos físico-químicos e sensoriais.

\section{MATERIAL E MÉTODOS}

Os frutos de mamão e cajá foram adquiridos em feiras livres da cidade de Belém - PA, no estágio de maturaçáo adequado para consumo ou processamento, no período de janeiro a fevereiro de 2007. Os hidrocolóides utilizados para as formulaçóes das frutas estruturadas foram: pectina de baixa metoxilação (CP Kelko, Limeira, Brasil), gelatina 180 Bloom (Rebiére, Amparo, Brasil) e alginato de sódio (Vetec, Duque de Caxias, Brasil). Os coadjuvantes tecnológicos utilizados no processo de estruturação das polpas foram: fosfato de cálcio bibásico $\left(\mathrm{CaHPO}_{4}\right)$ (Vetec, Duque de Caxias, Brasil), glicerina $\left(\mathrm{C}_{3} \mathrm{H}_{5}(\mathrm{OH})_{3}\right)$ (Vetec, Duque de Caxias, Brasil) e sacarose refinada comercial (União, São Paulo, Brasil).

Inicialmente, realizou-se uma seleção para escolha dos frutos sadios, rejeitando-se aqueles com podridóes e outros defeitos que poderiam comprometer a qualidade do produto final. Os frutos selecionados foram submetidos à lavagem em água corrente e em seguida sanitizados por imersão em solução aquosa de hipoclorito de sódio. Para o mamão a concentração da solução foi de $100 \mathrm{mg} \mathrm{L}^{-1}$ e imersão durante 20 minutos; para o cajá $30 \mathrm{mg} \mathrm{L}^{-1}$ durante 20 minutos. Posteriormente, foi realizado o enxágue dos frutos em solução de hipoclorito de sódio a $5 \mathrm{mg} \mathrm{L}^{-1}$. A sanitização dos equipamentos e utensílios utilizados foi realizada com solução de hipoclorito de sódio a $200 \mathrm{mg} \mathrm{L}^{-1}$.

Os frutos de cajazeira foram submetidos ao despolpamento em extrator de pás da marca Itametal (Itabuna, Brasil), modelo bonina 0.2 , com peneira de diâmetro equivalente a $1 \mathrm{~mm}$ de abertura de malha. $\mathrm{O}$ despolpamento dos mamóes foi realizado manualmente com auxílio de faca e colher de aço inoxidável, sendo a polpa triturada em liquidificador. As polpas obtidas não foram submetidas a tratamento térmico.

As polpas de mamão e cajá foram caracterizadas quanto ao pH (AOAC 1997), acidez titulável (AOAC 1997), sólidos solúveis (AOAC 1997), atividade de água (medição direta em analisador de atividade de água marca Decagon, modelo Pawkit, Pullman, EUA), umidade (AOAC 1997), cinzas (AOAC 1997), proteínas (AOAC 1997), lipídios (Bligh e Dyer 1959), fibras (método de detergência, segundo Goering e Van Soest 1970), açúcares totais e redutores (por Lane e Eynon (titulação de oxi-redução), segundo AOAC 1984), carboidratos (calculado pela diferença entre 100 e a soma das porcentagens de água, proteína, lipídeos totais e cinzas), taninos (extração segundo Bispo (1989) e quantificação pelo método colorimétrico de Folin-Denis no 952.03 da AOAC (1997)) e carotenóides totais (Godoy e Rodriguez-Amaya 1994).

Foram elaborados os estruturados mistos de cajá e mamão, na proporção de 1:1 (polpa cajá: polpa mamão), testando-se diferentes combinaçóes de alginato $\left(\mathrm{X}_{1}\right)$, pectina 
de baixa metoxilação $\left(\mathrm{X}_{2}\right)$ e gelatina $\left(\mathrm{X}_{3}\right)$, otimizados através de delineamento fatorial completo $2^{3}$, com 3 pontos centrais e 6 axiais (Neto et al. 1995) tendo como respostas: atividade de água $\left(\mathrm{Y}_{1}\right)$, firmeza $\left(\mathrm{Y}_{2}\right)$, sólidos solúveis $\left(\mathrm{Y}_{3}\right)$ e $\mathrm{pH}\left(\mathrm{Y}_{4}\right)$. A proporçấo de polpa de cajá e mamáo (1:1) foi mantida constante para todos os experimentos. Os níveis das variáveis estudadas no planejamento experimental foram definidos em função de dados da literatura (Grizotto et al. 2005a; Grizotto et al. 2005b) e testes preliminares realizados inicialmente, apresentados na Tabela 1.

Tabela 1 - Níveis das variáveis estudadas no planejamento experimental.

\begin{tabular}{lccccc}
\hline \multirow{2}{*}{$\begin{array}{l}\text { Variáveis } \\
\text { independentes }\end{array}$} & \multicolumn{5}{c}{ Níveis codificados das variáveis independentes } \\
\cline { 2 - 6 } & $-1,68$ & -1 & 0 & +1 & $+1,68$ \\
\cline { 2 - 6 } & \multicolumn{5}{c}{ Níveis reais das variáveis independentes } \\
\hline $\begin{array}{l}\text { Alginato }\left(\mathrm{g} \mathrm{kg}^{-1} \text { de }\right. \\
\text { polpa) }\end{array}$ & 1,6 & 5,0 & 10,0 & 15,0 & 18,4 \\
$\begin{array}{l}\text { Pectina B.M. (g } \\
\mathrm{kg}^{-1} \text { de polpa) }\end{array}$ & 3,2 & 5,0 & 17,5 & 30,0 & 36,8 \\
$\begin{array}{l}\text { Gelatina (g kg-1 de } \\
\text { polpa) }\end{array}$ & 16,0 & 50,0 & 100,0 & 150,0 & 184,0 \\
\hline
\end{tabular}

Todas as respostas do planejamento experimental (firmeza $\left(\mathrm{Y}_{1}\right)$, sólidos solúveis $\left(\mathrm{Y}_{2}\right), \mathrm{pH}\left(\mathrm{Y}_{3}\right)$ e atividade de água $\left(\mathrm{Y}_{4}\right)$ ) foram analisadas de acordo com Neto et al. (1995) com o auxílio do software Statistica ${ }^{\circledR}$ versão 5 (Tulsa, USDA). A firmeza dos estruturados foi determinada por mediçâo direta em reômetro da marca Sun Rheo Tex, modelo SD-700 (Tóquio, Japão), utilizando-se haste esférica de $5 \mathrm{~mm}$ de diâmetro, fixando-se a distância percorrida em $4,5 \mathrm{~mm}$, sendo os resultados expressos em grama (g). As demais análises foram realizadas de acordo com os métodos descritos anteriormente.

O processamento dos estruturados foi realizado de acordo com a metodologia proposta por Grizotto et al. (2005b), descrita a seguir. À polpa mista, na proporção de 1:1 (cajá:mamão) foi adicionado glicerol, na concentração de $100 \mathrm{~g} \mathrm{~kg}^{-1}$ de polpa e, em função do teor de sólidos solúveis, calculou-se a quantidade de sacarose suficiente para elevar seu teor para 50 BBrix. Em seguida a mistura foi aquecida a $60{ }^{\circ} \mathrm{C}$, adicionando-se o fosfato de cálcio e os hidrocolóides sob vigorosa agitação, utilizando-se misturador em hélice (Tecnal, Turratec TE-102, Piracicaba, Brasil) com rotação a $20000 \mathrm{rpm}$ por cerca de 5 minutos. Para a moldagem dos estruturados utilizou-se placas de petri de $15 \times 140 \mathrm{~mm}$, as quais foram mantidas sob refrigeração $\left(10^{\circ} \mathrm{C} / 24 \mathrm{~h}\right)$. A seguir, os estruturados foram cortados com auxílio de cortador de aço inoxidável. As frutas estruturadas, com dimensóes de $15 \mathrm{~mm}$ de diâmetro e $10 \mathrm{~mm}$ de altura, foram submetidas à secagem em estufa com circulação de ar a $45^{\circ} \mathrm{C}$ durante 8 horas, em seguida armazenadas em bandejas de poliestireno expandido e recobertas com filme de PVC.
Selecionaram-se, através dos resultados para a variável firmeza, obtidos do planejamento experimental, duas formulaçóes de estruturado, as quais foram analisadas sensorialmente. A avaliação sensorial foi realizada por 40 provadores não treinados, entre funcionários, visitantes e estagiários da Embrapa Amazônia Oriental. Empregou-se o teste de aceitaçáo com escala hedônica estruturada de 9 pontos ( 9 = gostei muitíssimo; 1 = desgostei muitíssimo) (Stone e Sidel 1993). As amostras foram apresentadas monadicamente aos potenciais consumidores à temperatura ambiente, em pratos plásticos descartáveis, codificados com números aleatórios de três dígitos e avaliadas quanto à textura, sabor, impressão global e intenção de compra. A análise de variância e o teste de Tukey foram realizados para a comparação das notas médias dadas pelos provadores na análise sensorial, através do software SAS 8.0 (Statistical Analysis System 1999), de modo a identificar a formulação de estruturado mais aceita pelos potenciais consumidores.

A formulação de estruturado misto de mamão e cajá selecionada através da avaliação sensorial foi analisada quanto à composição centesimal, firmeza, $\mathrm{pH}$, acidez titulável, sólidos solúveis, açúcares totais e redutores, atividade de água, carboidratos, valor energético total, taninos e carotenóides totais, de acordo com as metodologias citadas anteriormente. Para o cálculo de carotenóides totais do estruturado misto de cajá e mamão, empregou-se os valores de absorbância no comprimento de onda do licopeno (carotenóide principal do mamáo) e da $\beta$-criptoxantina (carotenóide principal do cajá), calculando-se a média dos valores encontrados e expressandose o resultado de carotenóides totais em $\mu \mathrm{g} \mathrm{g}^{-1}$.

\section{RESULTADOS E DISCUSSÃO}

Os resultados da caracterização físico-química das polpas de cajá e mamão estão apresentados na Tabela 2.

Os valores encontrados para a caracterização da polpa de cajá (Tabela 2) estão de acordo com a literatura consultada (Silva et al. 1999; Dias et al. 2003; Mattietto 2005). De acordo com a literatura, o $\mathrm{pH}$ pode variar de 2,1 a 3,3, o teor de sólidos solúveis de 8,80 a $12,3{ }^{\circ} \mathrm{B}$ e acidez titulável de 1,28 a 1,65\% em ácido cítrico. Mattietto (2005), em estudo sobre a polpa de cajá, observou $90,74 \%$ de umidade, $1,15 \%$ de proteína, $0,45 \%$ de lipídeos e $0,99 \%$ de cinzas.

$\mathrm{O}$ valor de carotenóides de $26,46 \mu \mathrm{g} \mathrm{g}^{-1}$ obtido para a polpa de cajá, está de acordo com os teores relatados na

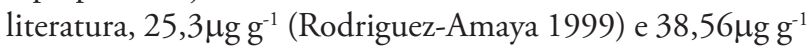
(Mattietto 2005). Para as análises de açúcares totais, redutores e não redutores os resultados estão próximos aos encontrados na literatura (Barbosa et al. 1981; Da Silva et al. 1997; Dias 2003). Para o teor de taninos, o valor observado $\left(1,50 \mathrm{mg} \mathrm{g}^{-1}\right)$ está próximo ao citado por Silva et al. (1999), de 1,64 $\mathrm{mg} \mathrm{g}^{-1}$ de polpa in natura. 
Tabela 2 - Caracterização físico-química das polpas de cajá e mamão.

\begin{tabular}{|c|c|c|}
\hline Determinação & Cajá & Mamão \\
\hline $\mathrm{pH}$ & $2,28 \pm 0,01$ & $5,01 \pm 0,06$ \\
\hline Atividade de água & $0,98 \pm 0,01$ & $0,99 \pm 0,00$ \\
\hline Sólidos solúveis ( $\left.{ }^{\circ} B r i x\right)$ & $9,35 \pm 0,06$ & $13,27 \pm 0,06$ \\
\hline Acidez titulável (\% ác. cítrico) & $1,62 \pm 0,03$ & $0,13 \pm 0,00$ \\
\hline Umidade (\%) & $89,23 \pm 0,01$ & $88,50 \pm 0,25$ \\
\hline Cinzas (\%)* & $3,73 \pm 0,06$ & $2,78 \pm 0,07$ \\
\hline Fibras $(\%)^{*}$ & $3,22 \pm 0,07$ & $3,52 \pm 0,08$ \\
\hline Proteínas (\%)* & $1,48 \pm 0,07$ & $1,06 \pm 0,03$ \\
\hline Lipídeos (\%)* & $1,16 \pm 0,08$ & $1,13 \pm 0,12$ \\
\hline Carboidratos (\%) & $10,09 \pm 0,06$ & $10,93 \pm 0,12$ \\
\hline Açúcares totais $\left(\mathrm{g} \mathrm{kg}^{-1}\right)$ & $76,20 \pm 0,72$ & $101,90 \pm 0,15$ \\
\hline Açúcares redutores $\left(\mathrm{g} \mathrm{kg}^{-1}\right)$ & $74,90 \pm 1,18$ & $93,23 \pm 0,75$ \\
\hline Açúcares não redutores $\left(\mathrm{g} \mathrm{kg}^{-1}\right)$ & $1,32 \pm 0,01$ & $8,33 \pm 0,63$ \\
\hline Carotenóides totais $\left(\mu \mathrm{g} \mathrm{g}^{-1}\right)$ & $26,46 \pm 4,61$ & $24,67 \pm 0,56$ \\
\hline Taninos $\left(\mathrm{mg} \mathrm{g}^{-1}\right)$ & $1,53 \pm 0,12$ & $0,83 \pm 0,09$ \\
\hline
\end{tabular}

*Resultados em base seca.

Para a polpa de mamáo, os valores encontrados estáo próximos ao relatado na literatura, com valores variando de 5,20 a 5,71 para o $\mathrm{pH}$, sólidos solúveis de 9,81 a 12,3B, acidez titulável de 0,04 a 0,16\% em ácido cítrico e umidade média de 85,42\% (Almeida et al. 2006; Rocha et al. 2007; Taco 2007). Os resultados de açúcares totais, redutores e não redutores, estão ligeiramente superiores aos relatados por Rocha et al. (2007), que encontraram 8,09\% de açúcares redutores e $0,28 \%$ para açúcares não redutores para mamão Formosa 'Tainung 01'. Para carotenóides totais, o valor encontrado de $24,67 \mu \mathrm{g} \mathrm{g}^{-1}$, está de acordo com a faixa citada por Sentanin e Amaya (2007) estudando mamóes de três cultivares provenientes da Bahia e do Espírito Santo.

$\mathrm{Na}$ Tabela 3 são apresentados os resultados de firmeza, sólidos solúveis, $\mathrm{pH}$ e atividade de água, obtidos dos experimentos com estruturados mistos de cajá e mamão, de acordo com a matriz do planejamento experimental.

De acordo com os resultados da análise estatística obtidos para a resposta firmeza, observou-se que somente o efeito gelatina linear foi significativo a $95 \%$ de confiança. A partir desse resultado, efetuou-se a análise de variância (ANOVA) obtendo-se valor de $\mathrm{r}^{2}$ de 0,74 , o que indica que $74 \%$ da variabilidade na resposta da firmeza pode ser explicada pelo modelo. A relação para a regressão $\left(\mathrm{MQ}_{\text {Regressão }}\left(\mathrm{MQ}_{\text {Residuo }}\right)\right.$ apresentou um valor de 9,52 e o valor de $\mathrm{F}_{\text {Calculado }}$ (resíduo) igual a 0,93 foi inferior ao $\mathrm{F}_{\text {Tabelado }}(2,53)$ para o mesmo, fornecendo uma baixa relação. A falta de ajuste do modelo foi baixa com $\mathrm{F}_{\text {Calculado }}$ de 2,06, sendo bem menor que o $\mathrm{F}_{\text {Tabelado }}$ $(19,42)$, sinalizando assim um modelo válido e útil para fins preditivos, sendo apresentado a seguir:

$\mathrm{Y}=360,38+317,30 \mathrm{xG}$

em que: $Y=$ firmeza; $G$ = gelatina

$\mathrm{O}$ efeito significativo da gelatina sobre a firmeza dos estruturados pode ser mais facilmente visualizado na Figura 1.

Através do gráfico de superfície e contorno, observa-se que na medida em que se aumenta a concentraçáo de gelatina, independente do aumento da concentraçáo dos demais hidrocolóides, aumenta também a firmeza dos estruturados.

Tabela 3 - Resultados dos experimentos do planejamento fatorial completo para as variáveis firmeza, sólidos solúveis, pH e atividade de água para estruturado misto de cajá e mamão.

\begin{tabular}{|c|c|c|c|c|c|c|c|}
\hline \multirow{2}{*}{ Experimento } & \multicolumn{3}{|c|}{ Níveis das variáveis em unidades reais $\left(\mathrm{g} \mathrm{kg}^{-1}\right)$} & \multicolumn{4}{|c|}{ Respostas* } \\
\hline & Alginato & Pectina & Gelatina & Firmeza $(\mathrm{g})$ & SST ( ${ }^{\circ}$ Brix) & $\mathrm{pH}$ & Atividade de água \\
\hline 1 & 5 & 5 & 50 & 20,80 & 73,67 & 4,18 & 0,60 \\
\hline 2 & 15 & 5 & 50 & 48,00 & 71,00 & 4,41 & 0,67 \\
\hline 3 & 5 & 30 & 50 & 54,00 & 73,00 & 4,28 & 0,67 \\
\hline 4 & 15 & 30 & 50 & 134,44 & 75,33 & 4,41 & 0,68 \\
\hline 5 & 5 & 5 & 150 & 679,60 & 66,67 & 4,49 & 0,62 \\
\hline 6 & 15 & 5 & 150 & 884,20 & 64,33 & 4,64 & 0,66 \\
\hline 7 & 5 & 30 & 150 & 1103,20 & 61,33 & 4,57 & 0,68 \\
\hline 8 & 15 & 30 & 150 & 807,60 & 57,67 & 4,73 & 0,67 \\
\hline 9 & 10 & 15 & 100 & 364,80 & 79,67 & 4,55 & 0,56 \\
\hline 10 & 10 & 15 & 100 & 308,20 & 81,67 & 5,54 & 0,58 \\
\hline 11 & 10 & 15 & 100 & 119,40 & 77,67 & 4,53 & 0,73 \\
\hline 12 & 1,6 & 15 & 100 & 134,00 & 73,33 & 4,33 & 0,69 \\
\hline 13 & 18,4 & 15 & 100 & 283,20 & 70,67 & 4,57 & 0,75 \\
\hline 14 & 10 & 3,2 & 100 & 210,60 & 65,67 & 4,44 & 0,75 \\
\hline 15 & 10 & 36,8 & 100 & 294,40 & 63,33 & 4,46 & 0,75 \\
\hline 16 & 10 & 15 & 16 & 9,00 & 55,00 & 4,33 & 0,89 \\
\hline 17 & 10 & 15 & 184 & 671,00 & 47,66 & 4,60 & 0,75 \\
\hline
\end{tabular}

* Média de 3 replicatas. Somente para a firmeza o valor representa a média de 7 replicatas. 


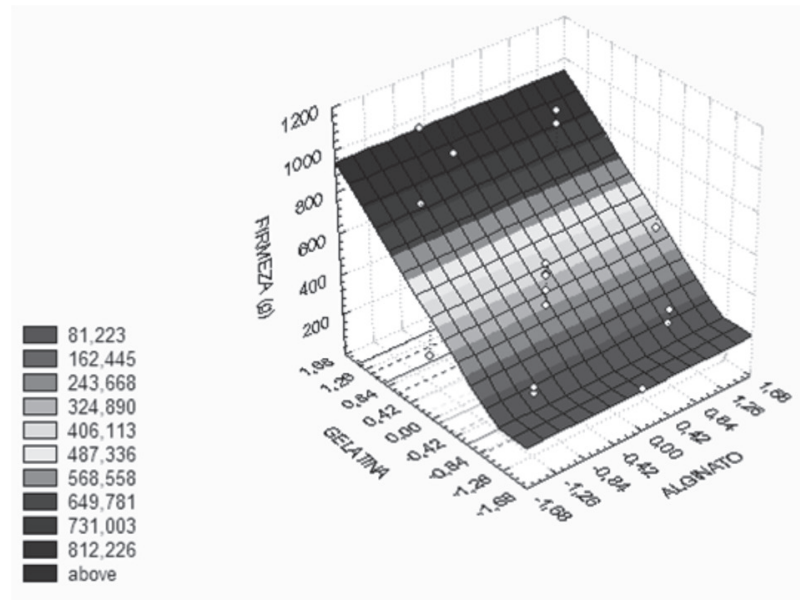

Figura 1 - Gráfico de superfície de resposta para a variável firmeza.

Com os resultados obtidos na análise do teor de sólidos solúveis (Tabela 3), realizou-se a análise estatística dos dados observando-se efeito significativo para as variáveis pectina quadrática, gelatina linear e gelatina quadrática, a um intervalo de confiança de 95\%. A partir das variáveis significativas, efetuou-se a análise de variância (ANOVA). Os valores para o $\mathrm{r}^{2}, \mathrm{~F}_{\text {Tabelado }}(3,13,95)$ regressão e $\mathrm{F}_{\text {Tabelado }}(13,11,95)$ resíduo foram respectivamente $0,87,3,41$ e 2,78 . O valor de $r^{2}$ indica que $87 \%$ da variabilidade na resposta é explicada pelo modelo. A relação $\mathrm{F}_{\text {Calculado }} / \mathrm{F}_{\text {Tabelado }}$ para a regressão apresentou valor de 5,59, concluindo-se que o ajuste do modelo é bom, podendo ser utilizado para fins preditivos. $\mathrm{O}$ valor de $\mathrm{F}_{\text {Calculado }}$ para a falta de ajuste $(5,49)$ foi menor que o $\mathrm{F}_{\text {Tabelado }}$ para o mesmo $(16,405)$, fornecendo uma baixa relação, o que é ideal na validação de um modelo. A equação apresentada a seguir, descreve o modelo codificado.

$\mathrm{Y}=79,25-3,96 \times \mathrm{P}^{2}-4,05 \mathrm{xG}-8,62 \mathrm{xG}^{2}$

onde: $\mathrm{Y}=$ sólidos solúveis; $\mathrm{P}=$ pectina; $\mathrm{G}=$ gelatina

O gráfico de superfície de resposta é apresentado na Figura 2.

Através do gráfico, nota-se que o uso da concentração de pectina e gelatina próximo aos níveis centrais promove um incremento no teor de sólidos solúveis do produto (faixa vermelha de maior intensidade) e que regiôes de mínimo são quase inexistentes.

De acordo com os resultados obtidos para a variável pH (Tabela 3), realizou-se a análise estatística dos dados observando-se como significativos a 95\% de confiança, os termos linear e quadrático das variáveis alginato $\left(\mathrm{X}_{1}\right)$, pectina $\left(\mathrm{X}_{2}\right)$ e gelatina $\left(\mathrm{X}_{3}\right)$. Porém, a partir da análise de variância das variáveis significativas, observou-se que a relação $\mathrm{MQ}_{\text {Regressão }}$ $\mathrm{MQ}_{\text {Resíduo }}\left(\mathrm{F}_{\text {Calculado }}\right.$ para regressão) apresentou valor de 1,49 , não

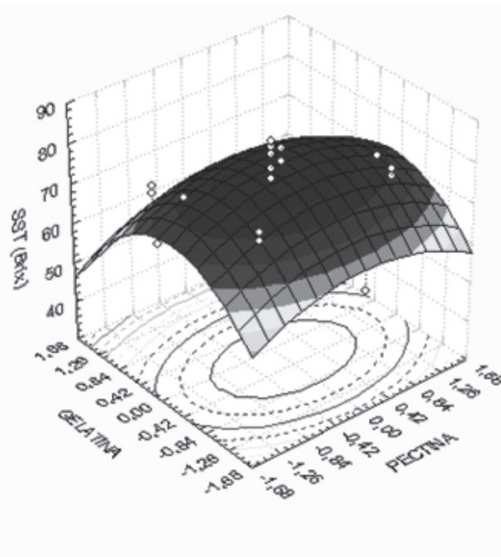

Figura 2 - Gráfico de superfície de resposta para a variável sólidos solúveis.

satisfazendo a exigência de ser pelo menos 3 vezes maior que o $\mathrm{F}_{\text {Tabelado }}(3,22)$ para que o modelo seja considerado válido e, portanto, não obteve-se um modelo válido para a variável $\mathrm{pH}$. Para a análise de atividade de água observou-se que os valores ajustados dos modelos linear e quadrático não apresentaram nenhum termo significativo a um intervalo de confiança de 95\%. Assim, qualquer variação nos valores de atividade de água pode ser atribuída somente ao erro experimental.

Através das respostas obtidas para a variável firmeza no planejamento experimental, selecionaram-se duas formulaçôes de estruturado misto de cajá e mamão e aplicaram-se os testes de aceitação e intenção de compra. As formulaçóes selecionadas foram F4 (1,5\% de alginato; 3\% de pectina e $5 \%$ de gelatina) e F15 ( $1 \%$ de alginato; $3,68 \%$ de pectina e $5 \%$ de gelatina), porém a F15 com $5 \%$ de gelatina e náo $10 \%$ como mostra a Tabela 3. Essa diminuição na concentração de gelatina para a F15 foi realizada em função dos valores de firmeza e da observação visual de uma boa estruturação das polpas. Essa duas formulaçóes avaliadas foram chamadas de Formulação A e Formulação B.

$\mathrm{Na}$ Tabela 4 observam-se as notas médias obtidas nos testes sensoriais para as duas formulaçôes de estruturados de cajá e mamão.

De acordo com os dados apresentados na Tabela 4, observa-se que para os estruturados mistos de cajá e mamáo, o teste de Tukey não identificou diferença estatística entre as formulaçốes estudadas (Formulação A e B). Porém, observa-se que a Formulação B apresentou médias ligeiramente superiores para os três atributos avaliados.

Quanto à intenção de compra (Figura 3), para as duas formulaçóes, observa-se grande número de provadores opinando com notas nos níveis 4 ou 5, ou seja, "possivelmente compraria" ou "certamente compraria", confirmando a impressáo positiva dos produtos pelos potenciais consumidores. 
Tabela 4 - Médias das notas obtidas para o teste de aceitação das formulações de estruturados mistos de cajá e mamão e suas respectivas \% de aceitação.

\begin{tabular}{lcccccc}
\hline \multirow{2}{*}{ Estruturado } & \multicolumn{2}{c}{ Textura } & \multicolumn{2}{c}{ Sabor } & \multicolumn{2}{c}{ Impressão Global } \\
\cline { 2 - 8 } & Médias & $\%$ aceit. & Médias & $\%$ aceit. & Médias & $\%$ aceit. \\
\hline Formulação A & $7,29 \pm 1,60 \mathrm{a}$ & 81,00 & $7,38 \pm 1,51 \mathrm{a}$ & 82,00 & $7,29 \pm 1,11 \mathrm{a}$ & 81,00 \\
Formulação B & $7,44 \pm 1,12 \mathrm{a}$ & 82,66 & $7,54 \pm 1,34 \mathrm{a}$ & 83,77 & $7,37 \pm 1,11 \mathrm{a}$ & 81,89 \\
\hline
\end{tabular}

Médias ( $n=40$ ) com letras iguais, em uma mesma coluna, não diferem entre si pelo Teste de Tukey, a $5 \%$ de probabilidade.

Formulação $A=1,5 \%$ de alginato; $3 \%$ de pectina e $5 \%$ de gelatina e Formulação $B=1 \%$ de alginato; $3,68 \%$ de pectina e $5 \%$ de gelatina.

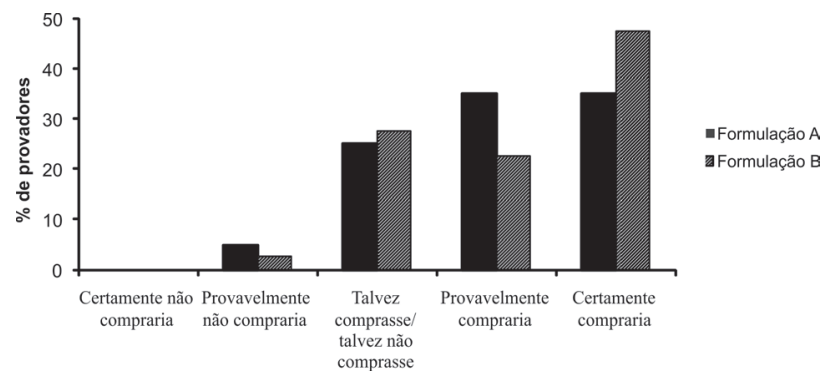

Figura 3 - Intenção de compra das formulações de estruturados mistos de cajá e mamão.

Observa-se ainda, através do histograma da Figura 3, que a Formulação B de estruturado misto se destacou no nível de certeza dos provadores, com relação à intenção de compra, com 31 provadores confirmando que "possivelmente" ou "certamente" comprariam o produto se o encontrassem à venda.

Assim, de acordo com os resultados da análise sensorial, selecionou-se a Formulação B de estruturado misto de cajá e mamão para a caracterização físico-química.

Os resultados da caracterização da formulação de estruturado misto de cajá e mamão, selecionada através da análise sensorial, encontram-se na Tabela 5 .

Tabela 5 - Caracterização físico-química do estruturado misto de cajá e mamão, em base úmida.

\begin{tabular}{lc}
\hline Determinação & Estruturado de cajá e mamão \\
\hline Firmeza (g) & $186,14 \pm 27,39$ \\
\hline Atividade de água & $0,77 \pm 0,01$ \\
pH & $4,21 \pm 0,03$ \\
\hline Sólidos solúveis ('Brix) & $70,30 \pm 0,48$ \\
\hline Acidez titulável (\% ác. cítrico) & $0,06 \pm 0,00$ \\
\hline Umidade (\%) & $39,95 \pm 2,31$ \\
Cinzas (\%) & $1,61 \pm 0,04$ \\
Fibras (\%) & $1,00 \pm 0,01$ \\
Proteínas (\%) & $9,16 \pm 0,09$ \\
\hline Lipídeos (\%) & $0,12 \pm 0,01$ \\
Carboidratos (\%) & $49,16 \pm 0,61$ \\
Valor energético $\left(\mathrm{Kcal} \mathrm{kg}^{-1}\right)$ & $2343,60 \pm 2,47$ \\
Açúcares totais $\left(\mathrm{g} \mathrm{kg}^{-1}\right)$ & $465,22 \pm 2,10$ \\
Açúcares redutores $\left(\mathrm{g} \mathrm{kg}^{-1}\right)$ & $278,45 \pm 6,72$ \\
Açúcares não redutores $\left(\mathrm{g} \mathrm{kg}^{-1}\right)$ & $174,12 \pm 2,03$ \\
Carotenóides totais $\left(\mu \mathrm{g} \mathrm{g}^{-1}\right)$ & $9,31 \pm 0,28$ \\
Taninos (mg g-1) & $0,19 \pm 0,00$ \\
\hline
\end{tabular}

Os resultados de firmeza representam a média de sete determinações. As demais análises foram realizadas em triplicata.
O estruturado misto de cajá e mamão é um produto novo, portanto não se encontrou referência na literatura sobre a caracterização físico-química do mesmo. Entretanto, ressaltase que os valores apresentados na Tabela 5 estão de acordo com resultados encontrados para outros estruturados de frutas (Freitas 1999; Grizotto et al. 2005a; Grizotto et al. 2005b).

Com relação à firmeza, o valor encontrado para o estruturado misto de cajá e mamão (Tabela 5) está próximo ao relatado por Grizotto et al. (2005b) ao estudar o estruturado de polpa concentrada de abacaxi, com valores entre 38,16 e $416,39 \mathrm{~g}$.

O valor de atividade de água de 0,77 , obtido para o estruturado misto de cajá e mamão, é inferior ao citado por Grizotto et al. (2005b), estudando estruturados de polpa concentrada de abacaxi, de manga e de mamão. Já Santos (2003), encontrou 0,583 de atividade de água em estudo sobre estruturado de manga. A atividade de água indica a quantidade de água disponível para facilitar a ocorrência de transformaçôes bioquímicas ou para o crescimento das células microbianas nos alimentos. Com atividade de água (Aa) de 0,77 observada neste trabalho, podemos classificar os estruturados obtidos como alimentos de Aa intermediária. Os alimentos com teor intermediário de água apresentam níveis de umidade entre 20 e $50 \%$ e $0,60 \leq \mathrm{Aa} \leq 0,85$ e, por isto, estáo sujeitos a processos de deterioração provocados, principalmente, por bolores e leveduras (Uboldi Eiroa 1981).

Quanto à análise de carotenóides totais, observa-se que, embora tenham ocorrido perdas durante o processamento, o valor encontrado para o estruturado misto de cajá e mamão, $9,31 \mu \mathrm{g} \mathrm{g}^{-1}$, é bastante significativo quando comparado aos teores observados para outras frutas in natura, como pêssego $\left(6,9 \mu \mathrm{g} \mathrm{g}^{-1}\right)$, manga $\left(12,8 \mu \mathrm{g} \mathrm{g}^{-1}\right)$ e maracujá $\left(4,7 \mu \mathrm{g} \mathrm{g}^{-1}\right)$ (Godoy e Rodriguez-Amaya 1994). O mesmo foi observado quanto ao teor de tanino, verificando-se valores significativos, mesmo após o processo de estruturação das frutas.

\section{CONCLUSÕES}

Os resultados obtidos através do planejamento experimental indicam que somente o aumento da concentração de gelatina afeta a firmeza do produto final.

A secagem dos estruturados a $45^{\circ} \mathrm{C} / 8 \mathrm{~h}$ promoveu redução no teor de umidade para níveis de umidade intermediária e minimizou o problema de adesividade na superfície das frutas, 
proporcionando maior estabilidade e melhoria da textura do produto final.

Embora ocorram perdas durante o processamento dos estruturados, os valores observados para os teores de carotenóides totais e taninos ainda são bastante significativos quando comparados aos teores observados para outras frutas in natura.

Os estruturados de frutas desenvolvidos apresentaram 81,0 e $81,9 \%$ de aceitaçáo em relaçáo à impressão global, para a formulação 1 e formulação 2, respectivamente. Para a intenção de compra, $70 \%$ dos provadores manifestaram interesse em comprar as formulaçôes estudadas, se as encontrassem à venda.

\section{BIBLIOGRAFIA CITADA}

Almeida, R.F.; Martins, M.L.L.; Resende, E. D.; Vitorazi, L.; Carlos, L.A.; Pinto, L.K.A. 2006. Influence of the refrigerating temperature on the chemical characteristics of the papaya fruits cv "Golden". Ciência e Tecnologia de Alimentos, 26(3): 577-581 (in Portuguese, with abstract in English).

AOAC. 1984. Official methods of analysis of the Association of Official Analytical Chemists. 14 ed. Arlington, VA, USA.

AOAC. 1997. Official methods of analysis of the Association of Official Analytical Chemists. 16 ed. Washington: Horwitz, W.

Barbosa, W.C.; Nazaré, R.F.R.; Hashimoto, K. 1981. Bromatological and technological study of the graviola and caja. Belém, EMBRAPA. Boletim de Pesquisa, 32. 15 pp (in Portuguese).

Bispo, E.S. 1989. Studies of products industrially of umbu (Spondias tuberosa, Arr. Câmara). Dissertação de Mestrado, Faculdade de Engenharia de Alimentos, Universidade Federal do Ceará, Fortaleza, Ceará. 119 pp. (in Portuguese).

Bligh, E.G; Dyer, W.J. 1959. A rapid method of total lipid and purification. Canadian Journal of Biochemistry and Physiology, 7: 911-917.

Bosco, J.; Soares, K.T.; Aguiar Filho, S.P.; Barros, R.V. 2000. The cultivation of caja. João Pessoa, EMEPA, Paraíba. 29 pp. (in Portuguese).

Da Silva, A.P.V.; Maia, G.A.; Oliveira, G.S.F.; Figueiredo, R.W.; Brasil, I.M. 1997. Quality characteristics of pulpy caja fruit juice (Spondias lutea L.) obtained by enzymatic-mechanical extration. Ciência e Tecnologia de Alimentos, 17(3): 233-236. (in Portuguese, with abstract in English).

Dias, D.R.; Schwan, R.F.; Lima, L.C.O. 2003. Methodology for elaboration of fermented alcoholic beverage from yellow mombin (Spondias mombin). Ciência e Tecnologia de Alimentos, 23(3): 342-350 236. (in Portuguese, with abstract in English).

Freitas, S.M.L. 1999. Use of sodium alginate in textured mix of orange juice and carrot energy-reduced. Dissertação de Mestrado em Tecnologia de Alimentos, Faculdade de Engenharia de Alimentos, Universidade Estadual de Campinas, Campinas, São Paulo, 110 pp. (in Portuguese).
Godoy, H.T.; Rodriguez-Amaya, D.B. 1994. Occurrence of cisisomers of provitamin A in Brazilian fruits. Journal of Agricultural and Food Chemistry, 42: 1306-1313.

Goering, H.K.; Van Soest, P.J. 1970. Forage Fiber Analyses: Apparatus, reagents, procedures and some applications. Washington, USDA/ Agricultural Research Service. p. 19.

Grizotto, R.K.; Bruns, R.E.; Aguirre, J. M.; Batista, G. 2005a. Optimization by the surface methodology of thecnological parameters for the production of restructured and dried fruit made from concentrated papaya pulp. Ciência e Tecnologia de Alimentos, 25(1): 158-164 (in Portuguese, with abstract in English).

Grizotto, R.K.; Aguirre, J.M.; Menezes, H.C. 2005b. Restructured fruits of intermediate moisture from concentrated pineapple, mango and papaya pulps. Ciência e Tecnologia de Alimentos, 25(4): 691-697. (in Portuguese, with abstract in English).

Mattietto, R.A. 2005. Technological study of a mixed nectar of caja (Spondias lutea L.) and umbu (Spondias tuberosa, Arruda Câmara). Tese de Doutorado em Tecnologia de Alimentos, Faculdade de Engenharia de Alimentos, Universidade Estadual de Campinas, Campinas, São Paulo. 299 pp. (in Portuguese).

Morley, R.C; Sharma, C. 1986. Dietary fiber food products and methods of manufacture. US patent, 4: 565 .

Neto, B.B.; Scarminio, I. S.; Bruns, R.E. 1995. Planning and Optimization Experiments. 2 ed. Campinas, Editora da UNICAMP. 299 pp. (in Portuguese).

Rocha, R.H.C.; Menezes, J.B.; Nascimento, S.R.C.; Nunes, G.H.S. 2007. Quality of papaya 'Formosa' submitted to different refrigeration temperatures. Revista Caatinga, 20(1): 75-80. (in Portuguese, with abstract in English).

Rodriguez-Amaya, D.B. 1999. A guide to carotenoid analysis in foods. Washington, ILSI Press, 64 pp.

Sacramento, C.K.; Souza, F.X. 2000. Caja (Spondias mobin L.). Série Frutas Nativas, 4. Jaboticabal: Funep. 42 pp. (in Portuguese).

Santos, C.N.P. 2003. Developing a structured mango (Mangifera indica cv. Tommy Atkins) partially dehydrated by osmosis. Dissertação de Mestrado, Faculdade de Engenharia de Alimentos/Universidade Estadual de Campinas. Campinas, São Paulo. 80 pp. (in Portuguese).

Sentanin, M.A.; Amaya, D.B.R. 2007. Carotenoid levels in papaya and peach determined by high performance liquid chromatography. Ciência e Tecnologia de Alimentos, 27(1): 13-19 (in Portuguese, with abstract in English).

Silva, A.P.V.; Maia, G.A.; Oliveira, G.S.F.; Figueiredo, R.W.; Brasil, I.M. 1999. A study of the production of clarified caja fruit juice (Spondias lutea L.). Ciência e Tecnologia de Alimentos, 19(1): 3336 (in Portuguese, with abstract in English).

Stone, H.S.; Sidel, J.L. 1993. Sensory Evaluation Practies. 2ed. San Diego, Academic Press. 338 pp.

TACO. Brazilian Table of Food Composition. 2006. Versão II. 2ed. Campinas, SP, NEPA - UNICAMP. 113pp. (www.unicamp.br/ nepa/taco/). Acesso em 20/10/07 (in Portuguese). 
Tirapegui, J.; Castro, I.A. 2001. Tannins in human diets: functional aspects, p.253-265. In: De Angelis, R.C. Importance of plant foods in the protection of health: nutrition physiology of protective and preventive degenerative diseases. Editora Atheneu, São Paulo. (in Portuguese).

Uboldi Eiroa, M.N. 1981. Water activity: influence on the development of microorganisms and determination methods in food. Boletim do ITAL, 3(18): 353-383 (in Portuguese, with abstract in English).

Recebido em 23/11/2009

Aceito em 09/08/2010 